General model with experimental validation of electrical resonant frequency tuning of electromagnetic vibration energy harvesters

This article has been downloaded from IOPscience. Please scroll down to see the full text article.

2012 Smart Mater. Struct. 21105039

(http://iopscience.iop.org/0964-1726/21/10/105039)

View the table of contents for this issue, or go to the journal homepage for more

Download details:

IP Address: 152.78.66.4

The article was downloaded on 14/09/2012 at 09:40

Please note that terms and conditions apply. 


\title{
General model with experimental validation of electrical resonant frequency tuning of electromagnetic vibration energy harvesters
}

\author{
Dibin Zhu ${ }^{1}$, Stephen Roberts ${ }^{2}$, Thomas Mouille ${ }^{2}$, Michael J Tudor ${ }^{1}$ and \\ Stephen P Beeby ${ }^{1}$ \\ ${ }^{1}$ School of Electronics and Computer Science, University of Southampton, SO17 1BJ, UK \\ 2 Perpetuum Ltd, Epsilon House, Southampton Science Park, Southampton, SO16 7NS, UK \\ E-mail: dz@ecs.soton.ac.uk
}

Received 15 March 2012, in final form 8 August 2012

Published 7 September 2012

Online at stacks.iop.org/SMS/21/105039

\begin{abstract}
This paper presents a general model and its experimental validation for electrically tunable electromagnetic energy harvesters. Electrical tuning relies on the adjustment of the electrical load so that the maximum output power of the energy harvester occurs at a frequency which is different from the mechanical resonant frequency of the energy harvester. Theoretical analysis shows that for this approach to be feasible the electromagnetic vibration energy harvester's coupling factor must be maximized so that its resonant frequency can be tuned with the minimum decrease of output power. Two different-sized electromagnetic energy harvesters were built and tested to validate the model. Experimentally, the micro-scale energy harvester has a coupling factor of 0.0035 and an untuned resonant frequency of $70.05 \mathrm{~Hz}$. When excited at $30 \mathrm{mg}$, it was tuned by $0.23 \mathrm{~Hz}$ by changing its capacitive load from 0 to $4000 \mathrm{nF}$; its effective tuning range is $0.15 \mathrm{~Hz}$ for a capacitive load variation from 0 to $1500 \mathrm{nF}$. The macro-scale energy harvester has a coupling factor of 552.25 and an untuned resonant frequency of $95.1 \mathrm{~Hz}$ and $95.5 \mathrm{~Hz}$ when excited at $10 \mathrm{mg}$ and $25 \mathrm{mg}$, respectively. When excited at $10 \mathrm{mg}$, it was tuned by $3.8 \mathrm{~Hz}$ by changing its capacitive load from 0 to $1400 \mathrm{nF}$; it has an effective tuning range of $3.5 \mathrm{~Hz}$ for a capacitive load variation from 0 to $1200 \mathrm{nF}$. When excited at $25 \mathrm{mg}$, its resonant frequency was tuned by $4.2 \mathrm{~Hz}$ by changing its capacitive load from 0 to $1400 \mathrm{nF}$; it has an effective tuning range of about $5 \mathrm{~Hz}$. Experimental results were found to agree with the theoretical analysis to within $10 \%$.
\end{abstract}

(Some figures may appear in colour only in the online journal)

\section{Introduction}

With the development of low-power electronics and energy harvesting technology, self-powered systems have become a research hotspot over the past decade [1]. The main advantage of self-powered systems is that they offer very low, or even zero, maintenance, which makes them suitable for deployment in large-scale installations consisting of many (100s of) systems or in locations which are difficult to access or completely inaccessible following system installation. Therefore, the target of energy harvesting is to power autonomous 'fit and forget' electronic systems over their lifetime (1-25 years or more). Some possible energy sources for harvesting include photonic energy, thermal energy and mechanical energy (typically vibrations). This paper concerns vibration energy harvesters, which may be used as an alternative to or to augment solar and thermal energy harvesters depending on the presence and magnitude of the particular energy source in the application environment. 
The most common vibration energy harvesting solution is a linear energy harvester that converts kinetic energy to electrical energy using a transduction mechanism [2]. The linear vibration energy harvester produces a maximum output power when its resonant frequency matches the ambient vibration frequency. To generate maximum power from low-amplitude vibrations, linear vibration energy harvesters are typically designed to have a high $Q$-factor. If the ambient vibration frequency does not match the harvester's resonant frequency, the harvester's output power drops significantly due to the high $Q$-factor. To date, there are two ways to overcome this drawback. The first is to widen the bandwidth of the vibration energy harvester and the second is to tune the resonant frequency of the energy harvester to match the ambient vibration frequency [3].

Research has been presented on the first approach aimed at widening the bandwidth of a vibration energy harvester. One solution is an energy harvester array consisting of multiple energy harvesters each operating at different frequencies [4]. Thus, the assembled energy harvester array has a wide operational frequency range while the $Q$-factor can remain high. However, only one or few individual harvesters contribute to power output at a particular source frequency, depending on the bandwidth and frequency characteristic of the excitation vibration [3]. Therefore, this solution is not volume efficient in terms of generated power density. Non-linear energy harvesters [5] and harvesters with bi-stable structures $[6,7]$ are the two alternative solutions to increase the bandwidth of vibration energy harvesters. They can improve the performance of the energy harvester if the ambient vibration frequencies are at higher and lower frequency bands respectively relative to its resonant frequency and so their applicability depends on the specific application frequency spectrum and size constraints on the harvester. Furthermore, hysteresis in non-linear energy harvesters makes the output unpredictable since it is a function of the historical variation of the vibration frequency.

Alternative methods based on the second approach of tuning the resonant frequency include both mechanical and electrical methods. Ideally a closed-loop control system automatically tunes the resonant frequency, either mechanically or electrically, to match the ambient vibration frequency [8, 9], making long-term autonomous 'fit and forget' electronic systems feasible. Mechanical tuning requires a change of the mechanical properties of the energy harvester to tune its resonant frequency [10-13]. Electrical tuning requires resonant frequency tuning by adjusting the harvester's electrical load. In general the electrical approach consumes much less energy than the mechanical approach as it only involves selecting the electrical load via electronic switches compared with adjusting mechanical properties, which is more energy hungry if it can be achieved at all. Electrical tuning is hence much easier to implement than mechanical tuning.

The fundamental principle of the described electrical tuning method is to adjust its electrical load so that the maximum output power of the energy harvester occurs at a different frequency from the mechanical resonant frequency of the energy harvester. A detailed model of electrical resonant frequency tuning of piezoelectric vibration energy harvesters has been reported by Zhu et al [3]. Charnegie [14] presented a bimorph piezoelectric energy harvester whose resonant frequency can be adjusted by varying its load capacitance. The test results showed that if one piezoelectric layer was used for frequency tuning while the other was used for energy harvesting, the resonant frequency can be tuned by an average of $4 \mathrm{~Hz}$ with respect to the original frequency of $350 \mathrm{~Hz}$ by adjusting the load capacitance from 0 to $10 \mathrm{mF}$. If both layers were used for frequency tuning, the tuning range was an average of $6.5 \mathrm{~Hz}$ by adjusting the load capacitance by the same amount. However, the output power was reduced if both layers were used for frequency tuning, while if only one layer was used for frequency tuning the output power remained unchanged. An electrically tunable electromagnetic energy harvester was reported by Cammarano et al [15]. The resonant frequency of the energy harvester was tuned by separately adjusting resistive, capacitive and inductive loads. The tuning range was between 57.4 and $66.5 \mathrm{~Hz}$ with different combinations of the three electrical loads. Although electrical tuning of vibration electromagnetic energy harvesters has been experimentally demonstrated, a comprehensive model of the method is not available.

In this paper, a general model and detailed analysis of resonant frequency tuning of electromagnetic vibration energy harvesters by varying the capacitive load, is presented and verified by experiment for the first time. The model is derived in section 2. Section 3 summarizes guidelines in designing an electrically tunable electromagnetic energy harvester. One micro-scale and one macro-scale electromagnetic energy harvester have been tested and the results are compared with the analytical results to validate the theory in sections 4-6.

\section{Principles}

\subsection{Generic model of electrical tunable electromagnetic energy harvesters}

The motion of linear vibration electromagnetic energy harvesters can be described using a differential equation as [16]:

$$
m \frac{\mathrm{d}^{2} z(t)}{\mathrm{d} t^{2}}+b \frac{\mathrm{d} z(t)}{\mathrm{d} t}+k_{s} z(t)=-m \frac{\mathrm{d}^{2} y(t)}{\mathrm{d} t^{2}}
$$

where $m$ is the inertial mass, $b$ is the damping coefficient, $k_{\mathrm{S}}$ is the spring constant, $y(t)$ is the displacement of the housing and $z(t)$ is the relative motion of the inertial mass with respect to the housing. The form of equation (1) in the frequency domain can be obtained by taking a Laplace transform, which, when rearranged, becomes:

$$
-I(s)=E(s)\left(s C_{m}+\frac{1}{R_{m}}+\frac{1}{s L_{m}}\right)
$$

where $I(s)=\frac{m}{k_{\mathrm{e}}} s^{2} Y(s)=\frac{m a}{k_{\mathrm{e}}}$ is the input current and $E(s)=$ $k_{\mathrm{e}} s Z(s)$ is the induced voltage. $C_{m}=\frac{m}{k_{\mathrm{e}}^{2}}, R_{m}=\frac{k_{\mathrm{e}}^{2}}{b}, L_{m}=$ 


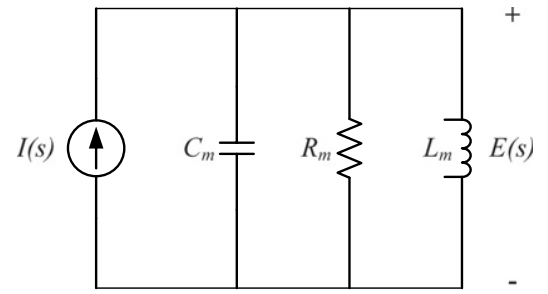

Figure 1. Equivalent circuit of an electromagnetic energy harvester.

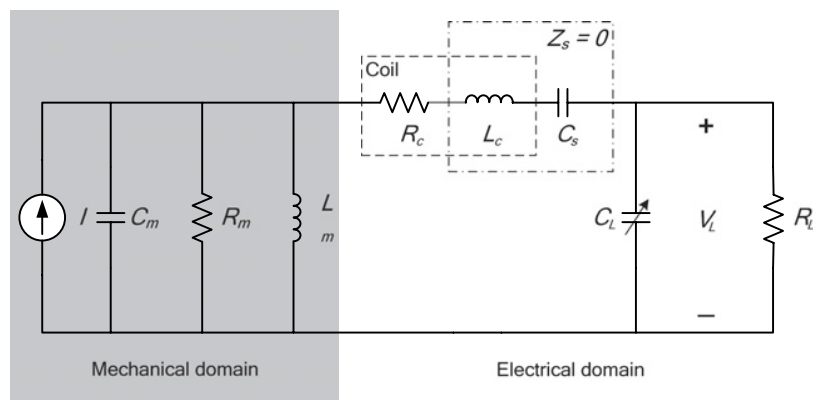

Figure 2. Equivalent circuit model of electromagnetic energy harvester with a variable capacitive load and a resistive load.

$\frac{k_{\mathrm{e}}^{2}}{k_{s}}$ are equivalent capacitance, resistance and inductance respectively. $a$ is the vibration acceleration and $k_{\mathrm{e}}$ is the electrical transduction constant, which is the ratio of the induced voltage to the velocity of the inertial mass. According to equation (2), an equivalent electrical circuit of a linear vibration energy harvester is shown in figure 1.

Figure 2 shows the equivalent circuit model of an electromagnetic energy harvester with a variable capacitive load, $C_{\mathrm{L}}$, and a resistive load, $R_{\mathrm{L}} \cdot R_{\mathrm{c}}$ is the coil resistance and $L_{\mathrm{c}}$ is the coil inductance.

The existence of the coil inductance increases the complexity of the circuit analysis. To simplify the analysis, a capacitor in series with the coil, $C_{\mathrm{s}}$, is used to cancel the effect of the coil inductance. Thus, the combined impedance of the coil inductance and the capacitor in series, $Z_{\mathrm{s}}$, should be zero, i.e.:

$$
Z_{s}=\frac{1}{\mathrm{j} \omega C_{s}}+\mathrm{j} \omega L_{\mathrm{c}}=0
$$

Therefore, the capacitor that is required to cancel the coil inductance is:

$$
C_{s}=\frac{1}{\omega^{2} L_{\mathrm{c}}} .
$$

If the impedance of the coil inductance is negligible compared to the coil resistance, the effect of the coil inductance can be ignored and no capacitor is needed for compensation.

Figure 3 shows the simplified equivalent circuit model after cancelling the effect of the coil inductance. The following analyses are based on this simplified model.

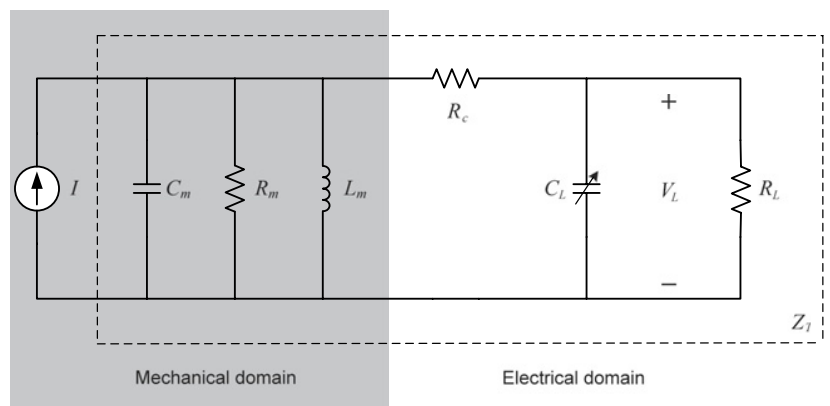

Figure 3. Simplified equivalent circuit model of an electromagnetic energy harvester with a variable capacitive load and a resistive load.

The total impedance of this circuit, $Z_{\mathrm{T}}$, is:

$$
Z_{\mathrm{T}}(\omega)=\left|\frac{1}{\mathrm{j} \omega C_{m}+\frac{1}{\mathrm{j} \omega L_{m}}+\frac{1}{R_{m}}+\frac{1}{R_{\mathrm{c}}+\frac{1}{\frac{1}{R_{\mathrm{L}}}+\mathrm{j} \omega C_{\mathrm{L}}}}}\right| .
$$

Therefore, the voltage across the capacitive load, $V_{\mathrm{L}}$, is given by:

$$
V_{\mathrm{L}}(\omega)=I\left|Z_{\mathrm{T}}(\omega) \frac{R_{\mathrm{L}} \| C_{\mathrm{L}}}{R_{\mathrm{c}}+R_{\mathrm{L}} \| C_{\mathrm{L}}}\right|
$$

where $R_{\mathrm{L}} \| C_{\mathrm{L}}=\frac{1}{\frac{1}{R_{\mathrm{L}}}+\mathrm{j} \omega C_{\mathrm{L}}}$, which is the combined impedance of $R_{\mathrm{L}}$ and $C_{\mathrm{L}}$ when they are connected in parallel.

$$
\begin{aligned}
V_{\mathrm{L}}(\omega)= & \frac{a m}{k_{\mathrm{e}}} \mid \frac{1}{\mathrm{j} \omega C_{m}+\frac{1}{\mathrm{j} \omega L_{m}}+\frac{1}{R_{m}}+\frac{1}{R_{\mathrm{c}}+\frac{1}{\frac{1}{R_{\mathrm{L}}}+\mathrm{j} \omega C_{\mathrm{L}}}}} \\
& \times \frac{\frac{1}{\frac{1}{R_{\mathrm{L}}}+\mathrm{j} \omega C_{\mathrm{L}}}}{R_{\mathrm{c}}+\frac{1}{\frac{1}{R_{\mathrm{L}}}+\mathrm{j} \omega C_{\mathrm{L}}}} \mid .
\end{aligned}
$$

The generated power across the resistive load, $P(\omega)$, is given by:

$$
\begin{aligned}
& P(\omega)= \frac{V_{\mathrm{L}}^{2}(\omega)}{R_{\mathrm{L}}} \\
& P(\omega)=\frac{a^{2} m^{2}}{k_{\mathrm{e}}^{2} R_{\mathrm{L}}}\left(\mid \frac{1}{\mathrm{j} \omega C_{m}+\frac{1}{\mathrm{j} \omega L_{m}}+\frac{1}{R_{m}}+\frac{1}{R_{\mathrm{c}}+\frac{1}{\frac{1}{R_{\mathrm{L}}}+\mathrm{j} \omega C_{\mathrm{L}}}}}\right. \\
&\left.\times \frac{\frac{1}{\frac{1}{R_{\mathrm{L}}}+\mathrm{j} \omega C_{\mathrm{L}}}}{R_{\mathrm{c}}+\frac{1}{\frac{1}{R_{\mathrm{L}}}+\mathrm{j} \omega C_{\mathrm{L}}}} \mid\right)^{2} .
\end{aligned}
$$

After rearranging equation (9), the equation to calculate the generated power across the resistive load becomes:

$$
\begin{aligned}
P(\omega)= & \frac{a^{2} K}{\omega^{2}} \Pi(\omega) \\
& \times \frac{1}{\left[\frac{\omega_{n}^{2}}{\omega^{2}}-1-\Sigma(\omega)\right]^{2}+\left[\frac{1}{Q}+\frac{K}{\omega m} \Theta(\omega)\right]^{2}}
\end{aligned}
$$




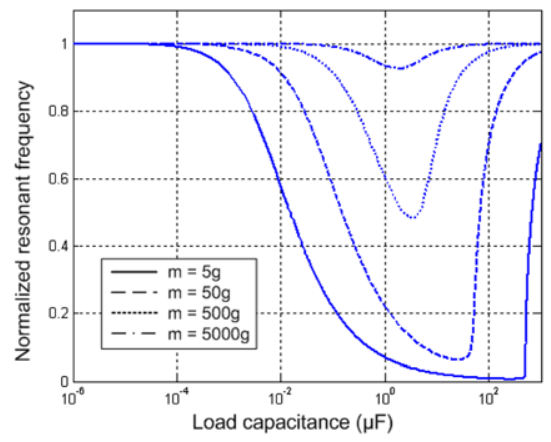

(a)

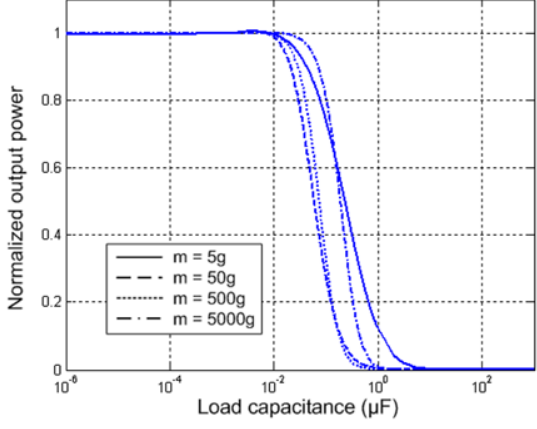

(b)

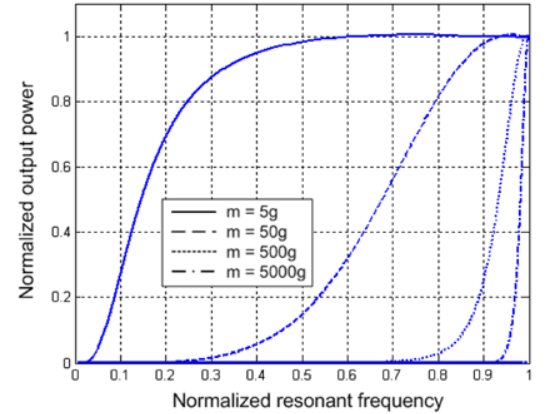

(c)

Figure 4. Effect of the inertial mass on the tuning range and output power of an electromagnetic energy harvester with a capacitive load $\left(R_{\mathrm{c}}=1000 \Omega, K=1000, r=1, Q=100\right)$. (a) $f_{\mathrm{r}}$ versus $C_{\mathrm{L}}$. (b) $P$ versus $C_{\mathrm{L}}$. (c) $P$ versus $f_{\mathrm{r}}$.

where $K=\frac{k_{\mathrm{e}}^{2}}{R_{\mathrm{c}}}$, is defined as the electromagnetic coupling factor, $r=\frac{R_{\mathrm{L}}}{R_{\mathrm{c}}}$, is the ratio of load resistance to the coil resistance, $\omega_{n}=\sqrt{\frac{k_{s}}{m}}$, is the original resonant frequency of the energy harvester, and $Q$ is the open circuit quality $(Q)$ factor of the energy harvester which is related to the mechanical damping of the energy harvester,

$$
\begin{aligned}
& \Pi(\omega)=\frac{r}{(1+r)^{2}+\omega^{2} R_{\mathrm{L}}^{2} C_{\mathrm{L}}^{2}}, \\
& \Sigma(\omega)=\frac{C_{\mathrm{L}} r^{2} \frac{K R_{\mathrm{c}}}{m}}{(1+r)^{2}+\omega^{2} R_{\mathrm{L}}^{2} C_{\mathrm{L}}^{2}}, \\
& \Theta(\omega)=\frac{(1+r)+\omega^{2} R_{\mathrm{L}}^{2} C_{\mathrm{L}}^{2}}{(1+r)^{2}+\omega^{2} R_{\mathrm{L}}^{2} C_{\mathrm{L}}^{2}} .
\end{aligned}
$$

Please note that $\Pi(\omega), \Sigma(\omega)$ and $\Theta(\omega)$ are used only to make the equation look concise. They do not have any physical meaning.

$P(\omega)$ reaches a maximum when

$$
\frac{\mathrm{d} P(\omega)}{\mathrm{d} \omega}=0
$$

i.e.

$$
\frac{\omega_{n}^{2}}{\omega^{2}}-1-\Sigma(\omega)=0
$$

Thus, the frequency at which the energy harvester produces maximum power, i.e. the new resonant frequency, $\omega_{\mathrm{r}}$, is obtained by solving equation (12) as:

$\omega_{\mathrm{r}}=\sqrt{\frac{\sqrt{\left(\theta+\chi-\varepsilon \omega_{n}^{2}\right)^{2}+4 \varepsilon \theta \omega_{n}^{2}}-\left(\theta+\chi-\varepsilon \omega_{n}^{2}\right)}{2 \varepsilon}}$

where $\theta=(1+r)^{2}, \chi=C_{\mathrm{L}} r^{2} \frac{K R_{\mathrm{c}}}{m}$ and $\varepsilon=R_{\mathrm{L}}^{2} C_{\mathrm{L}}^{2}$. Please note that these symbols are used only to make the equation look concise. They do not have any physical meaning.

The new resonant frequency, $\omega_{\mathrm{r}}$, is a function of the load capacitance, $C_{\mathrm{L}}$. Therefore, the resonant frequency can be tuned by adjusting the load capacitance.

When $\omega=\omega_{\mathrm{r}}$, the output power of the energy harvester is maximum. Substitution of equation (13) to (10) gives the equation for this maximum output power as:

$$
P_{\max }\left(\omega_{\mathrm{r}}\right)=\frac{a^{2} K}{\omega_{r}^{2}} \Pi\left(\omega_{\mathrm{r}}\right) \frac{1}{\left[\frac{1}{Q}+\frac{K}{\omega_{\mathrm{r}} m} \Theta\left(\omega_{\mathrm{r}}\right)\right]^{2}}
$$

where $\Pi\left(\omega_{\mathrm{r}}\right)$ and $\Theta\left(\omega_{\mathrm{r}}\right)$ are the same as those in equation (10), only with $\omega=\omega_{\mathrm{r}}$.

\subsection{Simulation results}

Figures 4, 5, 6, 7 and 8 show the simulation results of the model demonstrating the effect of the mass $(m), Q$-factor $(Q)$, the coil resistance $\left(R_{\mathrm{c}}\right)$, the electromagnetic coupling factor $(K)$ and the ratio of load resistance to coil resistance $(r)$ on the tuning range and output power of an electromagnetic energy harvester, respectively. In these figures, sub-figures (a) and (b) were obtained according to equations (13) and (14), respectively. Sub-figures (c) were drawn by combining respective sub-figures (a) and (b). In each simulation, the values of four of the five variables are fixed in order to determine the effect of the remaining variable. The values used in the simulation were chosen as examples and may vary in different energy harvesters. The untuned resonant frequency was set to be $100 \mathrm{~Hz}$. In all simulations, it was assumed that the energy harvester is excited under a sinusoidal vibration whose frequency always matches the resonant frequency of the energy harvester and that the acceleration level is $50 \mathrm{mg}\left(1 g=9.8 \mathrm{~m} \mathrm{~s}^{-2}\right)$. These values were chosen as indicative values as these parameters do not affect the tuning range or output power of the electromagnetic energy harvester. All simulation results of resonant frequency and output power were normalized to the resonant frequency and output power of the energy harvester without the tuning capacitor, respectively.

2.2.1. The effect of the mass $(m)$. Figure 4 shows the simulation results of how the mass of the energy harvester affects its tuning range and the output power. In this simulation, $R_{\mathrm{c}}, K, r$ and $Q$ are fixed to $1000 \Omega, 1000,1$ and 100 , respectively. It is found in figure 4(a) that, for the same $K$, the bigger the mass is, the smaller overall tuning range that can be achieved and the critical capacitive load. The 


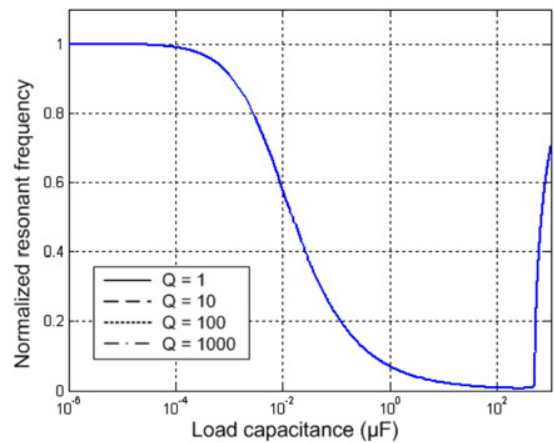

(a)

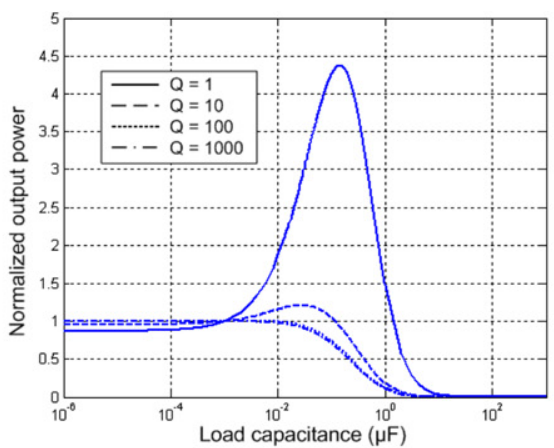

(b)

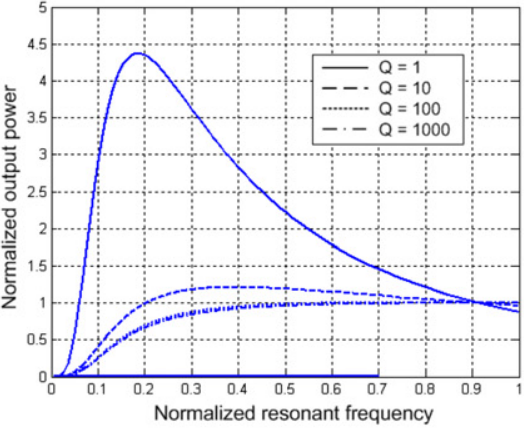

(c)

Figure 5. Effect of the $Q$-factor on the tuning range and output power of an electromagnetic energy harvester with a capacitive load $\left(R_{\mathrm{c}}=1000 \Omega, K=1000, r=1, m=5 \mathrm{~g}\right)$. (a) $f_{\mathrm{r}}$ versus $C_{\mathrm{L}}$. (b) $P$ versus $C_{\mathrm{L}}$. (c) $P$ versus $f_{\mathrm{r}}$.

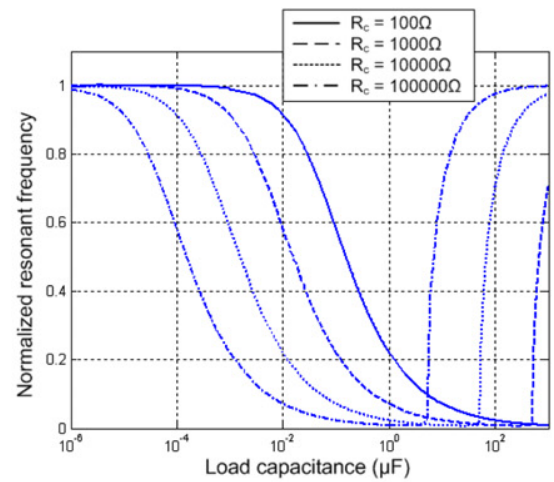

(a)

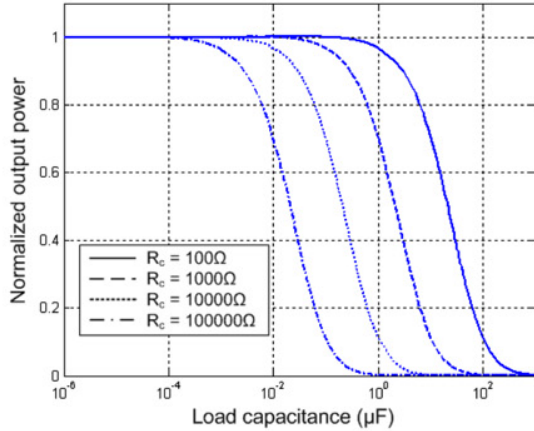

(b)

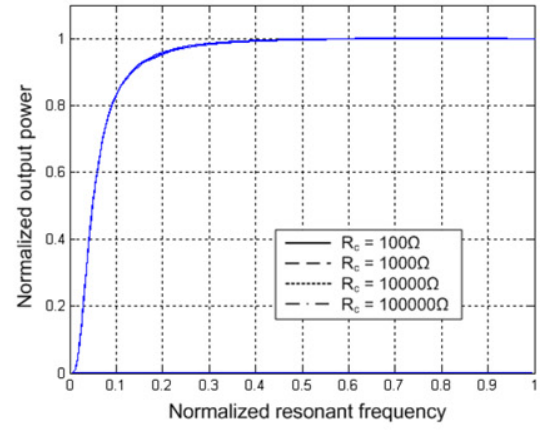

(c)

Figure 6. Effect of the coil resistance on the tuning range and output power of an electromagnetic energy harvester with a capacitive load ( $K=1000, r=1, m=5 \mathrm{~g}, Q=100)$. (a) $f_{\mathrm{r}}$ versus $C_{\mathrm{L}}$. (b) $P$ versus $C_{\mathrm{L}}$. (c) $P$ versus $f_{\mathrm{r}}$.

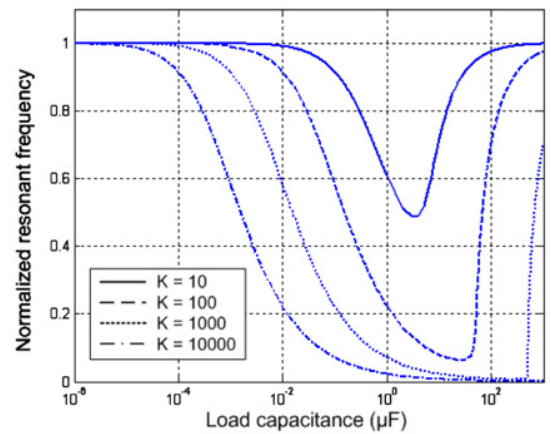

(a)

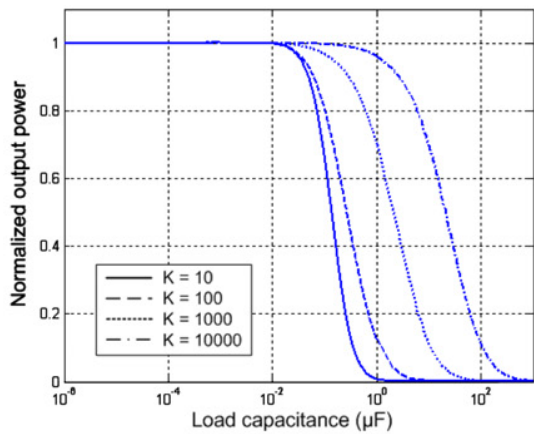

(b)

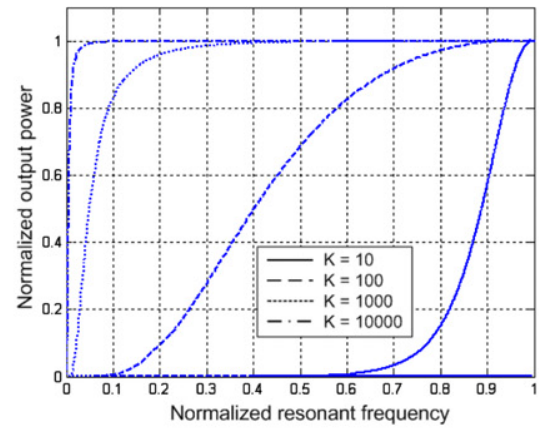

(c)

Figure 7. Effect of $K$ on the tuning range and output power of an electromagnetic energy harvester with a capacitive load $\left(R_{\mathrm{c}}=1000 \Omega, r=1, m=5 \mathrm{~g}, Q=100\right.$ ). (a) $f_{\mathrm{r}}$ versus $C_{\mathrm{L}}$. (b) $P$ versus $C_{\mathrm{L}}$. (c) $P$ versus $f_{\mathrm{r}}$.

critical capacitive load is defined as the capacitive load that tunes the resonant frequency to the lowest possible value. Figure 4(b) shows that the mass does not affect the value of the threshold capacitive load, which is defined as follows. If the load capacitance is smaller than the threshold capacitive load, the output power is higher than or equal to the output power without the capacitive load. If the load capacitance is higher than the threshold capacitive load, the output power starts to decrease to lower than the output power without the capacitive load. Figure 4(c) demonstrates that, the smaller the mass is, the larger the effective tuning range. The effective tuning range is defined as the tuning range across which the energy harvester generates at least half the output power level of an untuned energy harvester excited by the same excitation level at resonance. Therefore, the results suggest that, for the same $K$, the smaller the energy harvester is, the higher the effective tuning range that can be achieved.

2.2.2. The effect of the $Q$-factor $(Q)$. $\quad$ Figure 5 shows the effect of $Q$-factor of the energy harvester on its tuning range 


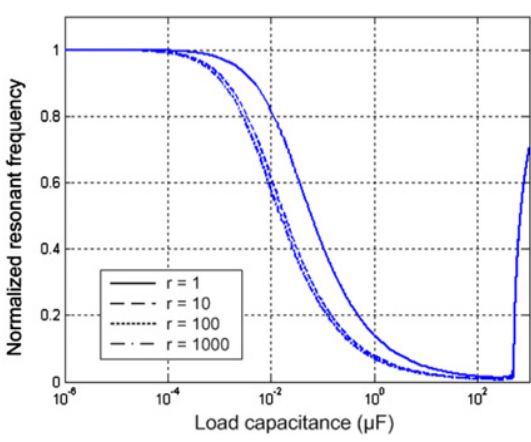

(a)

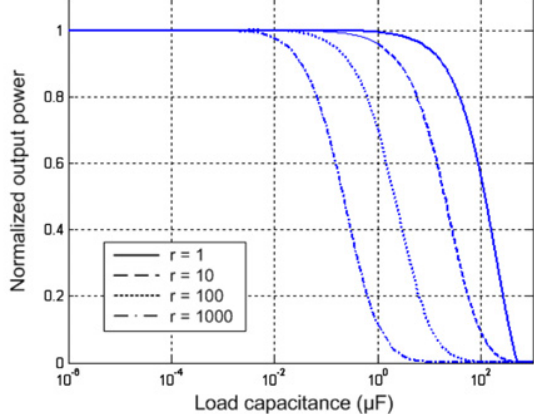

(b)

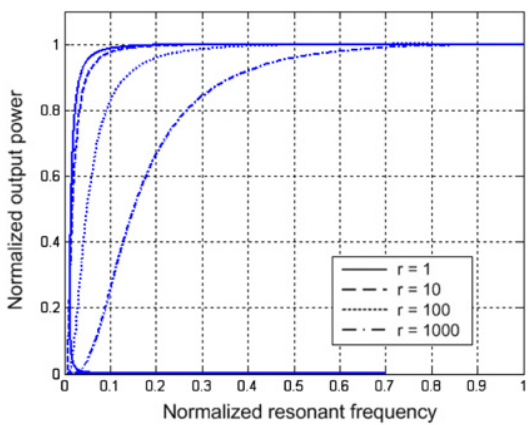

(c)

Figure 8. Effect of $r$ on the tuning range and output power of an electromagnetic energy harvester with a capacitive load $\left(R_{\mathrm{c}}=1000 \Omega\right.$, $K=1000, m=5 \mathrm{~g}, Q=100$ ). (a) $f_{\mathrm{r}}$ versus $C_{\mathrm{L}}$. (b) $P$ versus $C_{\mathrm{L}}$. (c) $P$ versus $f_{\mathrm{r}}$.

and the output power. In this simulation, $R_{\mathrm{c}}, K, r$ and $m$ are fixed to $1000 \Omega, 1000,1$ and $5 \mathrm{~g}$, respectively. It is found in figure 5 (a) that the $Q$-factor does not affect the overall tuning range of an electromagnetic energy harvester or the critical capacitive load. Figure 5(b) shows that the lower the $Q$-factor is, the higher threshold capacitive load will be. Figure 5(c) demonstrates that the lower the $Q$-factor, the larger the effective tuning range.

2.2.3. The effect of the coil resistance $\left(R_{\mathrm{c}}\right)$. Figure 6 shows the effect of coil resistance of the energy harvester on its tuning range and the output power. In this simulation, $K, r, m$ and $Q$ are fixed to $1000,1,5 \mathrm{~g}$ and 100 , respectively. It is found in figure 6(a) that the coil resistance does not affect the overall tuning range of an electromagnetic energy harvester but the critical capacitive load reduces with the increasing coil resistance. Figure 6(b) shows that, the higher the coil resistance, the lower the threshold capacitive load. Figure 6(c) demonstrates that the coil resistance does not affect the effective tuning range of the electromagnetic energy harvester. The simulation suggests that, the larger the coil resistance, the smaller the load capacitance that is needed to achieve resonant frequency tuning.

\subsubsection{The effect of the electromagnetic coupling factor $(K)$.} Figure 7 shows the effect of the electromagnetic coupling factor of the energy harvester on its tuning range and the output power. In this simulation, $R_{\mathrm{c}}, r, m$ and $Q$ are fixed to $1000 \Omega, 1,5 \mathrm{~g}$ and 100 , respectively. It is found in figure 7(a) that, the higher the electromagnetic coupling factor, the higher the overall tuning range of an electromagnetic energy harvester and the critical capacitive load. Figure 7(b) shows that, the higher the coil resistance, the higher the threshold capacitive load. Figure 7(c) demonstrates that the increasing electromagnetic coupling factor can increase the effective tuning range of the electromagnetic energy harvester. These results suggest that the electromagnetic coupling factor should be as high as possible to achieve the maximum effective tuning range.

2.2.5. The effect of the ratio of load resistance to coil resistance $(r)$. Figure 8 shows how the ratio of load resistance to coil resistance of the energy harvester affects its tuning range and the output power. In this simulation, $R_{\mathrm{c}}, K, m$ and $Q$ are fixed to $1000 \Omega, 1000,5 \mathrm{~g}$ and 100 , respectively. It is found in figure 8(a) that the load to coil resistance ratio does not affect the overall tuning range of an electromagnetic energy harvester or the critical capacitive load. However, the threshold capacitive load increases with decreasing load to coil resistance ratio as shown in figure 8(b). Figure 8(c) demonstrates that, the lower the load to coil resistance ratio, the larger the effective tuning range of the electromagnetic energy harvester. These results suggest that the load to coil resistance ratio should be as small as possible to achieve the maximum effective tuning range.

2.2.6. Summary. Simulation results suggest that the resonant frequency of the electromagnetic energy harvester decreases with increasing load capacitance until it reaches the critical capacitive load at which the resonant frequency is at its minimum. If the load capacitance is further increased, above the critical capacitive load, the resonant frequency will then increase with the load capacitance. However, the resonant frequency of the energy harvester can never exceed the original resonant frequency without a load capacitance.

Further, when the load capacitor is lower than the threshold capacitive load, the output power of the tuned energy harvester is the same as or greater than that of the untuned energy harvester. Conversely, when the load capacitance is higher than the threshold capacitive load, the output power decreases with capacitive load. The output power is completely damped to zero by the load capacitor when the load capacitance becomes very high. The values of the critical and the threshold capacitive loads depend on the design parameters of the energy harvester, as discussed in sections 2.2.1-2.2.5.

\subsection{The maximum output power}

In the cases when the value of load resistance is relatively high compared to the coil resistance (for example a rectifier), i.e. $R_{\mathrm{L}} \gg R_{\mathrm{c}}$ and thus $r \gg 1$, it is true that

$$
(1+r)^{2}>1+r \gg \omega_{r}^{2} R_{\mathrm{L}}^{2} C_{\mathrm{L}}^{2} .
$$


Thus,

$$
\begin{gathered}
(1+r)^{2}+\omega_{r}^{2} R_{\mathrm{L}}^{2} C_{\mathrm{L}}^{2} \approx(1+r)^{2} \\
\frac{(1+r)+\omega_{r}^{2} R_{\mathrm{L}}^{2} C_{\mathrm{L}}^{2}}{(1+r)^{2}+\omega_{r}^{2} R_{\mathrm{L}}^{2} C_{\mathrm{L}}^{2}} \approx \frac{1}{1+r} .
\end{gathered}
$$

Therefore, equation (10) can be simplified as:

$$
P_{\max }\left(\omega_{\mathrm{r}}\right)=\frac{a^{2} K}{\omega_{\mathrm{r}}^{2}} \frac{r}{(1+r)^{2}} \frac{1}{\left(\frac{1}{Q}+\frac{K}{\omega_{\mathrm{r}} m} \frac{1}{1+r}\right)^{2}} .
$$

Then, the maximum output power, $P_{\max }$, is

$$
P_{\max }=\frac{a^{2} K r m^{2}}{\left[\frac{\omega_{\mathrm{r}} m(1+r)}{Q}+K\right]^{2}} .
$$

Equation (19) shows that the maximum output power is a function of the resonant frequency. When the resonant frequency increases, the maximum power decreases. For a tunable vibration energy harvester, it is desirable that the power level does not change significantly with a change in the resonant frequency.

If the electromagnetic coupling factor, $K$, is so high that

$$
K \gg \frac{\omega_{\mathrm{r}} m(1+r)}{Q}
$$

the following equation stands:

$$
\frac{\omega_{\mathrm{r}} m(1+r)}{Q}+K \approx K
$$

Therefore, by simplifying equation (19), the maximum output power becomes:

$$
P_{\max }=\frac{a^{2} r m^{2}}{K}
$$

which is independent of the resonant frequency. Therefore, it is important for an electromagnetic energy harvester to have a high electromagnetic coupling factor to achieve a large effective tuning range.

\section{Electromagnetic coupling factor, $K$}

\subsection{Basic equations}

As defined in equation (10), the electromagnetic coupling factor, $K$, is given by:

$$
K=\frac{k_{\mathrm{e}}^{2}}{R_{\mathrm{c}}}
$$

where $k_{\mathrm{e}}=\frac{V}{v}$ and $R_{\mathrm{c}}$ is the coil resistance, $V$ is the induced voltage within the coil and $v$ is the velocity of the inertial mass when vibrating.

According to Faraday's law of induction, the induced voltage within the coil is:

$$
V=-N \frac{\mathrm{d} \varphi}{\mathrm{d} t}
$$

where $N$ is the number of turns of the coil and $\frac{\mathrm{d} \varphi}{\mathrm{d} t}$ is the magnetic flux gradient.
Therefore, equation (23) can be rewritten as:

$$
K=\frac{N^{2}\left(\frac{\mathrm{d} \varphi}{\mathrm{d} t}\right)^{2}}{R_{\mathrm{c}} v^{2}} .
$$

As $N$ and $R_{\mathrm{c}}$ only depend on the coil geometry and dimensions, the coil coefficient, $\gamma$, is defined as:

$$
\gamma=\frac{N^{2}}{R_{\mathrm{c}}}
$$

Equation (25) then becomes:

$$
K=\gamma \frac{\left(\frac{\mathrm{d} \varphi}{\mathrm{d} t}\right)^{2}}{v^{2}}=\gamma \frac{\left(\frac{\mathrm{d} \varphi}{\mathrm{d} z} \frac{\mathrm{d} z}{\mathrm{~d} t}\right)^{2}}{v^{2}} .
$$

As $v=\frac{\mathrm{d} z}{\mathrm{~d} t}$, equation (27) can be simplified as:

$$
K=\gamma\left(\frac{\mathrm{d} \varphi}{\mathrm{d} z}\right)^{2} .
$$

According to equation (25), the coil coefficient as well as the magnetic flux gradient through the coil must be as high as possible and the velocity of the inertial mass should be as low as possible to increase the electromagnetic coupling factor. In addition, according to equation (28), the change in magnetic flux per unit displacement of the inertial mass has to be as high as possible to achieve a high electromagnetic coupling factor.

\subsection{Relationship between the coil and the electromagnetic coupling factor}

The number of the turns of the coil can be calculated by:

$$
N=\frac{4 F_{\mathrm{c}}\left(R_{\mathrm{o}}-R_{\mathrm{i}}\right) t}{\pi d^{2}}
$$

where $F_{\mathrm{c}}$ is the coil fill factor, the ratio of the volume of conductor to the volume of the coil, $R_{\mathrm{O}}$ and $R_{\mathrm{i}}$ are the outer and inner radius of the coil, respectively. $t$ is the total thickness of the coil and $d$ is the diameter of the coil wire.

The coil resistance is given by:

$$
R_{\mathrm{c}}=\rho \frac{L_{\mathrm{c}}}{A}
$$

where $\rho$ is the electrical resistivity of the wire material, $L_{\mathrm{c}}$ is the total length of the coil wire and $A$ is the cross area of the wire. The total wire length is:

$$
L_{\mathrm{c}}=\frac{4 F_{\mathrm{c}}\left(R_{\mathrm{o}}^{2}-R_{\mathrm{i}}^{2}\right) t}{d^{2}} .
$$

The cross-sectional area of the wire is:

$$
A=\frac{\pi d^{2}}{4} .
$$

Substitution of equations (31) and (32) into (30) yields the coil resistance rewritten as:

$$
R_{\mathrm{c}}=\rho \frac{16 F_{\mathrm{c}}\left(R_{\mathrm{o}}^{2}-R_{\mathrm{i}}^{2}\right) t}{\pi d^{4}} .
$$




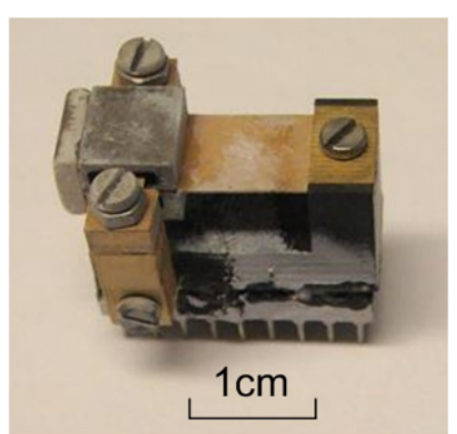

Figure 9. Overview of MiETEM.

Hence, the coil coefficient, $\gamma$, is:

$$
\gamma=\frac{N^{2}}{R_{\mathrm{c}}}=\frac{F_{\mathrm{c}}\left(R_{\mathrm{O}}-R_{\mathrm{i}}\right) t}{\rho \pi\left(R_{\mathrm{o}}+R_{\mathrm{i}}\right)} .
$$

Substitution of equation (34) into (28) gives the new equation of $K$ as

$$
K=\frac{F_{\mathrm{c}}\left(R_{\mathrm{o}}-R_{\mathrm{i}}\right) t}{\rho \pi\left(R_{\mathrm{o}}+R_{\mathrm{i}}\right)}\left(\frac{\mathrm{d} \phi}{\mathrm{d} z}\right)^{2} .
$$

Hence $K$ can be increased by increasing the coil fill factor and thickness and the ratio of the outer diameter of the coil to the inner diameter.

\subsection{Key points in designing electrically tunable electromagnetic energy harvester}

Based on the above analysis, the key points to design an electromagnetic energy harvester with a large effective tuning range are:

- the coupling factor, $K$, should be as large as possible;

- the bigger the energy harvester, the higher $K$ is needed to keep the output power of the energy harvester unchanged;
- the ratio of load resistance to coil resistance should be as low as possible;

- the $Q$-factor should be as low as possible;

- the coil resistance does not affect the effective tuning range of the electromagnetic energy harvester;

- the larger the coil resistance, the smaller the load capacitance needed to achieve frequency tuning;

- to achieve large $K$, the coil coefficient must be large, i.e. the coil must have a high fill factor and large thickness;

- the layout of magnets must be well designed so that the maximum change in flux linkage can be achieved with the minimum displacement of the magnets;

- with large $K$ and $r$, the inertial mass of the energy harvester must be large enough to ensure high output power.

\section{A micro-scale electrically tunable electromagnetic energy harvester}

\subsection{Overview}

Figure 9 shows a Micro-scale Electrically Tunable Electro Magnetic energy harvester (MiETEM).

Figure 10(a) shows the profile of MiETEM. It has a fixed coil and a resonator consisting of two magnets with magnetic flux guides mounted on a cantilever. Figure 10(b) shows the cross-sectional view of the $\mathrm{A}-\mathrm{A}^{\prime}$ plane in figure 8 . When the resonator vibrates, the magnets, together with the magnetic flux guide, move up and down relative to the fixed coil. The coil cuts the magnetic flux thus inducing a voltage across the coil.

The identical poles of the two magnets faced each other forming four magnetic flux paths. The magnets were $\mathrm{NdFeB}$, a strong permanent magnet. The magnetic flux guide was made of mild steel with a very high permeability. One piece of a $1 \mathrm{~mm}$ thick mild steel washer was placed between the two magnets to reduce the force between them and make the

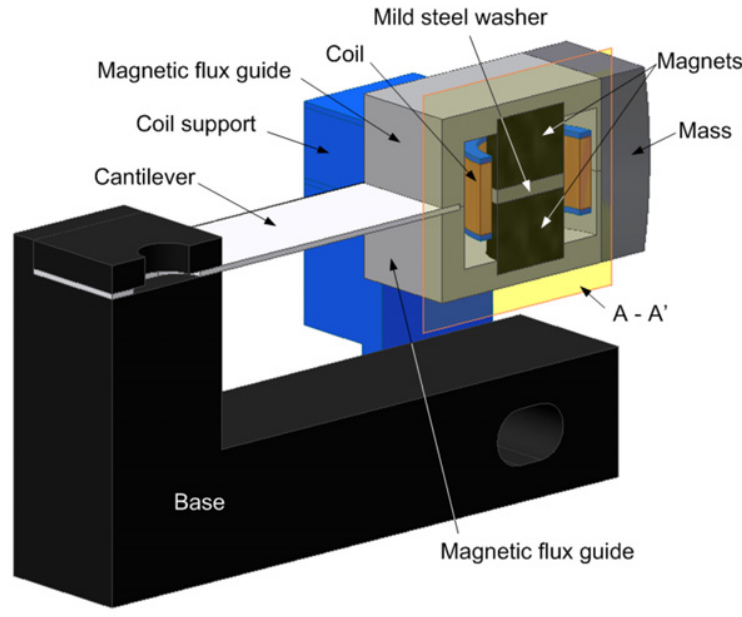

(a)

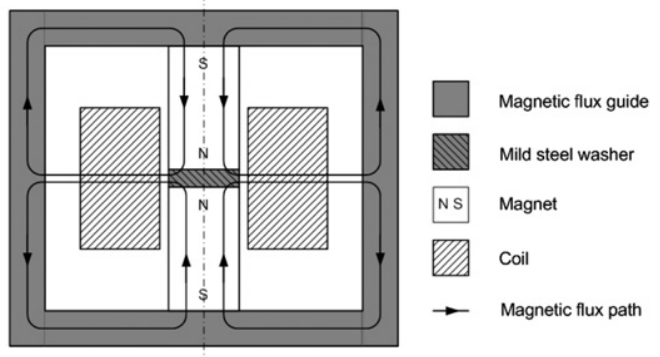

(b)

Figure 10. Structure of MiETEM. (a) Profile of MiETEM. (b) Cross-sectional view of MiETEM (A-A'). 


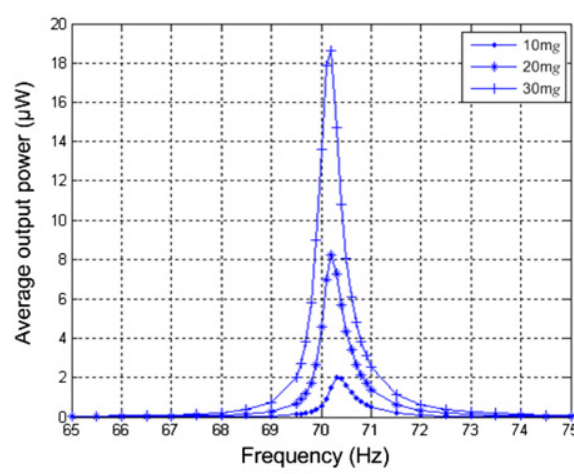

(a)

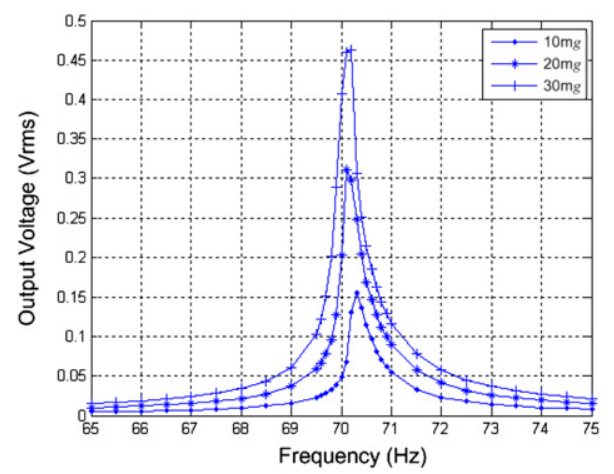

(b)

Figure 11. Performance of the untuned MiETEM. (a) Average output power at the optimum load. (b) Open circuit output RMS voltage.

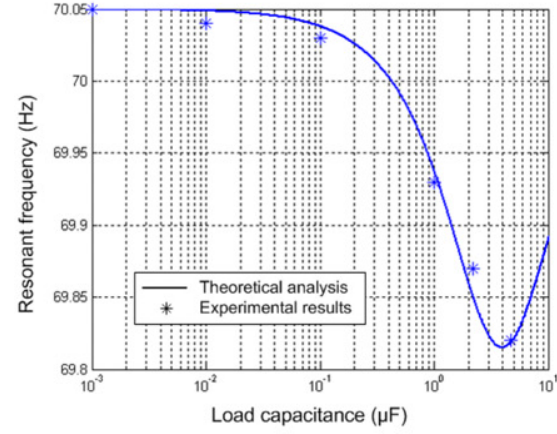

(a)

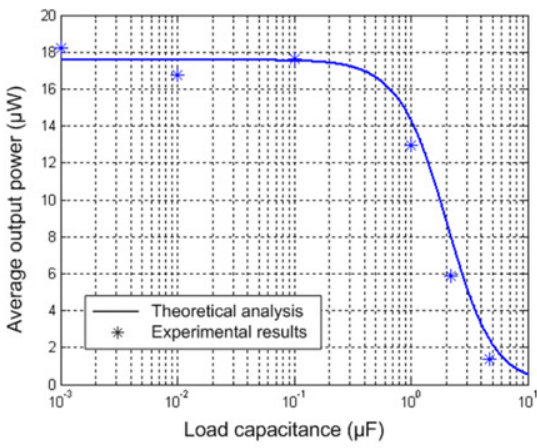

(b)

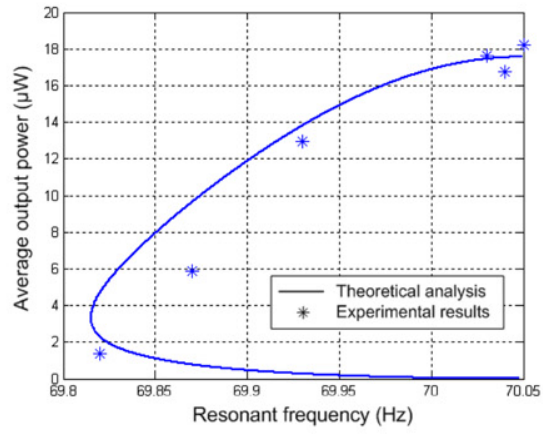

(c)

Figure 12. Tuning range and output power of MiETEM with variations of load capacitances. (a) $f_{\mathrm{r}}$ versus $C_{\mathrm{L}}$. (b) $P$ versus $C_{\mathrm{L}}$. (c) $P$ versus $f_{\mathrm{r}}$.

harvester easier to assemble and to divert the magnetic flux to the side magnetic flux guide, as shown in figure 10(b). The cantilever was made of $200 \mu \mathrm{m}$ thick BeCu.

The cylindrical coil was held by two coil supports attached to either side of the energy harvester. The outer and inner diameters of the coil were $5 \mathrm{~mm}$ and $4 \mathrm{~mm}$, respectively. The height of the coil was $2.5 \mathrm{~mm}$. The coil wire diameter was $25 \mu \mathrm{m}$. The coil resistance was measured as $697 \Omega$. The number of coil turns was around 1430 and the fill factor was 0.56 . In addition, the coil inductance was measured as $0.3 \mathrm{mH}$.

\subsection{Performance of MiETEM}

The total mass of the energy harvester was $5 \mathrm{~g}$ and the energy harvester had a resonant frequency of $70.05 \mathrm{~Hz}$ experimentally. The $Q$-factor of the energy harvester was measured as 120 and the optimum load resistance as $3700 \Omega$. The optimum load resistance is the load resistance on which the maximum output power is generated. The electromagnetic coupling factor was calculated to be 0.0035 according to equation (25).

Figure 11(a) shows the average power at the optimum resistive load and figure 11(b) the open circuit voltage of the untuned energy harvester as a function of excitation level and frequency. The maximum average output power was $18.4 \mu \mathrm{W}$ when the energy harvester was excited at $30 \mathrm{mg}$.

Figures 12(a) and (b) compare experiment and theory of the resonant frequency and maximum output power, respectively, with variation of load capacitance. The theoretical curves were drawn from equations (13) and (14), respectively. Combining figures 12(a) and (b) leads to figure 12(c), which shows the maximum output power as a function of resonant frequency. It is found the experimental resonant frequency and maximum output power agree with the theory to within $10 \%$. Experimentally, the overall tuning range is $0.23 \mathrm{~Hz}$ and the effective tuning range of the MiETEM is $0.15 \mathrm{~Hz}$. A capacitor was not required in series with the coil to cancel the coil inductance because the coil inductance is $0.3 \mathrm{mH}$, which, at and given the resonant frequency of $70.05 \mathrm{~Hz}$, gives an impedance of only $0.132 \Omega$ which is much smaller than the coil resistance of $697 \Omega$. Therefore, the effect of the coil inductance on the performance of the energy harvester in this case can be ignored. Furthermore, only load capacitances smaller than $10 \mu \mathrm{F}$ were used in the test. This is because when the load capacitance became large, the output voltage of the energy harvester was too small to detect. Table 1 summarizes the values used in equations (13) and (14) to obtain the theoretical curves in figure 12.

\section{A macro-scale electrically tunable electromagnetic energy harvester}

\subsection{Overview}

The Macro-scale Electrically Tunable Electro Magnetic energy harvester (MaETEM) described in this section was 
Table 1. Values used in equations (13) and (14) to obtain the theoretical curves in figure 12.

\begin{tabular}{llllllll}
\hline$K$ & $\begin{array}{l}\text { Untuned resonant } \\
\text { frequency (Hz) }\end{array}$ & $m(\mathrm{~g})$ & $Q$ & $R_{\mathrm{c}}(\Omega)$ & $R_{\mathrm{L}}(\Omega)$ & $r$ & $a(\mathrm{~m} g)$ \\
\hline 0.0035 & 70.05 & 5 & 120 & 697 & 3700 & 5.3 & 30 \\
\hline
\end{tabular}

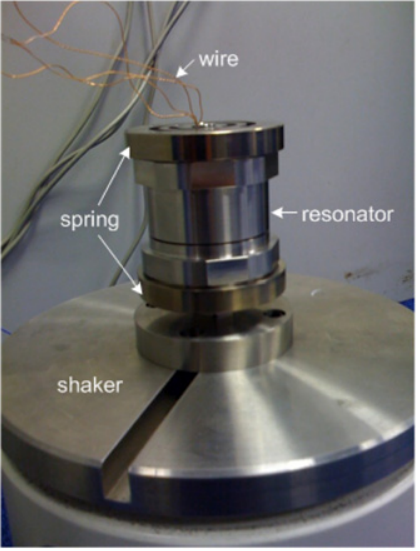

Figure 13. Photo of MaETEM (Courtesy of Perpetuum Ltd).

designed and built by Perpetuum Ltd. The energy harvester was a cylinder of which the diameter was $7 \mathrm{~cm}$ and the height $7.5 \mathrm{~cm}$, as shown in figure 13 . The total volume of the energy harvester was $289 \mathrm{~cm}^{3}$. Figure 14 shows the schematic cross-sectional view of the energy harvester along the centre axis. The energy harvester consisted of a resonator (as shown in figure 14(b)) and a stator (as shown in figure 14(c)).

During the operation, the centre rod was attached to the vibration source. The air gaps between the static magnetic flux guide and the movable magnetic flux guide, $g_{1}$ and $g_{2}$, vary with movement of the resonator, which changes the magnetic field flux through the two coils and thus induces voltage across the coils. Both air gaps were $0.2 \mathrm{~mm}$ when the energy harvester was static. As the displacement of the resonator is limited by these air gaps, the maximum displacement of the resonator is $0.2 \mathrm{~mm}$.

The magnet and the magnetic flux guide were made of hard and soft ferrite, respectively. The spring is made of stainless steel. Magnet and coil holders were made of plastic. The total mass of the energy harvester was $830 \mathrm{~g}$. The electromagnetic coupling factor was calculated to be 552.25, according to equation (25). The coil resistance and inductance were measured as $99.5 \Omega$ and $4 \mathrm{H}$, respectively.

\subsection{Performance of MaETEM}

The energy harvester was tested at an excitation level of $10 \mathrm{mg}$ and $25 \mathrm{mg}$. The resonant frequencies of the energy harvester were $95.1 \mathrm{~Hz}$ and $95.5 \mathrm{~Hz}$ when excited at $10 \mathrm{mg}$ and $5 \mathrm{mg}$, respectively. The $Q$-factor of the energy harvester was measured as 1350 when excited at $10 \mathrm{mg}$ and 1250 when excited at $25 \mathrm{mg}$. The difference in resonant frequency and $Q$-factor at different vibration accelerations is caused by slight non-linearity of the energy harvester due to the weak magnetic force between the stator and the resonator.

As the coil inductance was $4 \mathrm{H}$, and given the resonant frequencies of $95.1 \mathrm{~Hz}$ and $95.5 \mathrm{~Hz}$, the impedance of the coil inductance was $2390 \Omega$ and $2400 \Omega$ when excited at $10 \mathrm{mg}$ and $25 \mathrm{mg}$, respectively. They are much bigger than the coil resistance of $99.5 \Omega$. Therefore, a compensation capacitor needs to be connected in series with the coil to cancel the effect of the coil inductance. As shown in equation (4), the value of this compensation capacitor is frequency dependent, which makes selection of a single capacitor awkward. As an approximation, a capacitor of $680 \mathrm{nF}$, which is the single capacitor closest to the calculated value based on the untuned resonant frequency, was used in all tests described in this section.

5.2.1. Excited at $10 \mathrm{mg}$. Figure 15 shows the output power of the energy harvester with a resistive load of $10 \mathrm{k} \Omega$ and various capacitances ranging from 0 to $1400 \mathrm{nF}$, when excited at $10 \mathrm{mg}$. Note that $10 \mathrm{k} \Omega$ is not the optimum load resistance of the energy harvester. The purpose of using a designated resistor rather than the optimum load resistor is to validate the generality of the model.

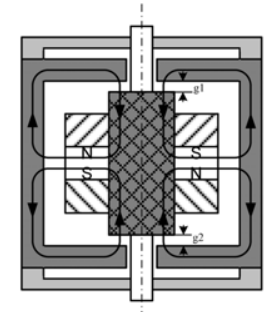

(a)

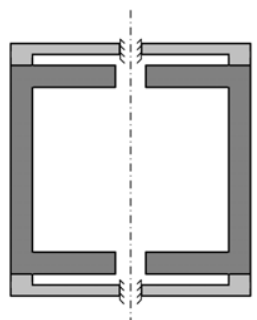

(b)

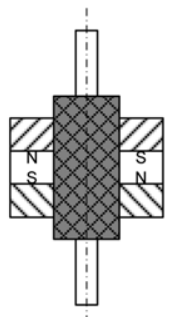

(c)

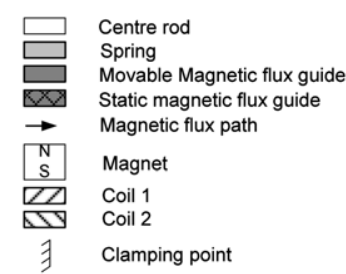

Figure 14. Schematic cross-sectional view of MaETEM. (a) Overall. (b) The resonator. (c) The stator. 
Table 2. Values used in equations (13) and (14) to obtain the theoretical curves in figure 16

\begin{tabular}{llllllll}
\hline$K$ & $\begin{array}{l}\text { Untuned resonant } \\
\text { frequency }(\mathrm{Hz})\end{array}$ & $m(\mathrm{~g})$ & $Q$ & $R_{\mathrm{c}}(\Omega)$ & $R_{\mathrm{L}}(\Omega)$ & $r$ & $a(\mathrm{~m} g)$ \\
\hline $\mathrm{T} \mathrm{m})$ & 830 & 1350 & 99.5 & 10000 & 100.5 & 10 \\
\hline 552.25 & 95.1 & & & & & &
\end{tabular}

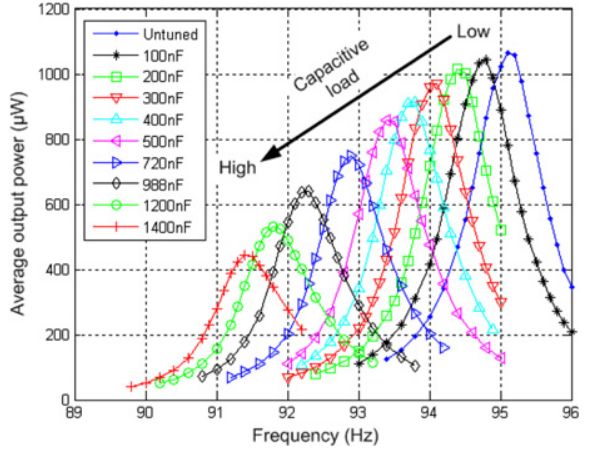

Figure 15. Output power of MaETEM with variations of load capacitances when excited at $10 \mathrm{mg}$ (load resistance is $10 \mathrm{k} \Omega$ ).

Figures 16(a) and (b) compare experiment and theory of the resonant frequency and the maximum output power variation with load capacitance. The theoretical curves were drawn according to equation (13) for resonant frequency and equation (14) for output power. Combining figures 16(a) and (b) leads to figure 16(c), which shows the maximum output power as a function of resonant frequency. The experimental results agree with the theory to within 5\%. The effective tuning range of the energy harvester is $3.5 \mathrm{~Hz}$. Like in the test of MiETEM, only load capacitances smaller than $10 \mu \mathrm{F}$ were used in this test. Table 2 summarizes the values used in equations (13) and (14) to obtain the theoretical curves in figure 16.

5.2.2. Excited at $25 \mathrm{mg}$. Figure 17 shows the power spectrum of MaETEM with a resistive load of $4 \mathrm{k} \Omega$ and various capacitances ranging from 0 to $1600 \mathrm{nF}$ when excited

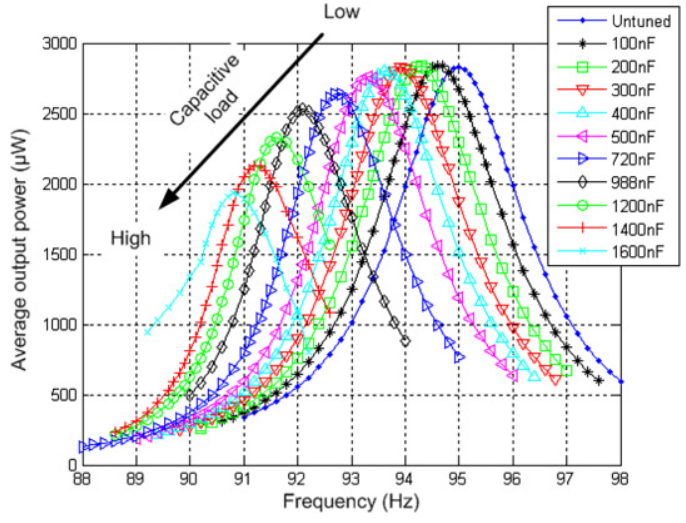

Figure 17. Output power of MaETEM with variations of load capacitances when excited at $25 \mathrm{mg}$ (load resistance is $4 \mathrm{k} \Omega$ ).

at $25 \mathrm{mg}$. Note again that $4 \mathrm{k} \Omega$ is not the optimum load resistance of the energy harvester.

Comparisons of experiment and theory of the resonant frequency and the maximum output power with variation of load capacitances are shown in figures 18(a) and (b), respectively. The theoretical curves are drawn according to equation (13) for resonant frequency and equation (14) for the maximum output power. Combining figures 18(a) and (b) leads to figure 18(c), which shows the maximum output power at various resonant frequencies. The effective tuning range of the energy harvester is over $5 \mathrm{~Hz}$. The experimental results agree with the theory to within $10 \%$. Table 3 summarizes the values used in equations (13) and (14) to obtain the theoretical curves in figure 18.

5.2.3. Discussions. It is found that the resonant frequency reduced with an increase of the load capacitance as

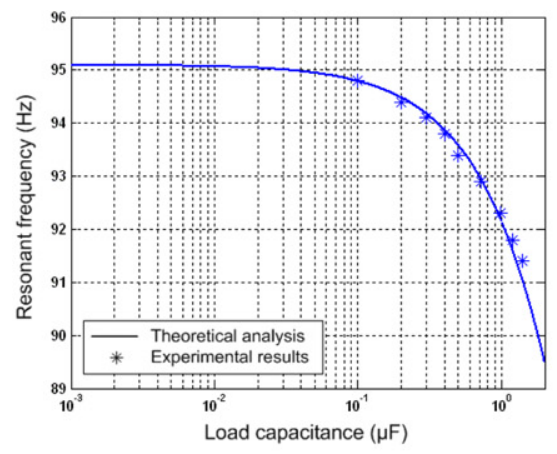

(a)

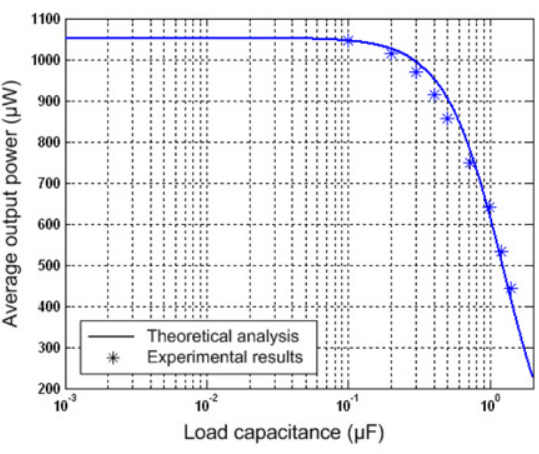

(b)

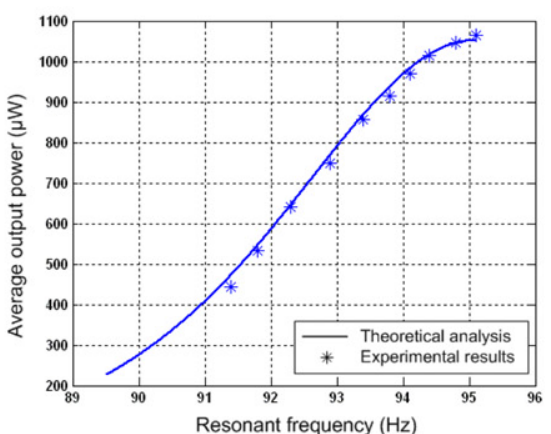

(c)

Figure 16. Tuning range and output power of MaETEM with variations of load capacitances (excited at $10 \mathrm{mg}$ ). (a) $f_{\mathrm{r}}$ versus $C_{\mathrm{L}}$. (b) $P$ versus $C_{\mathrm{L}}$. (c) $P$ versus $f_{\mathrm{r}}$. 


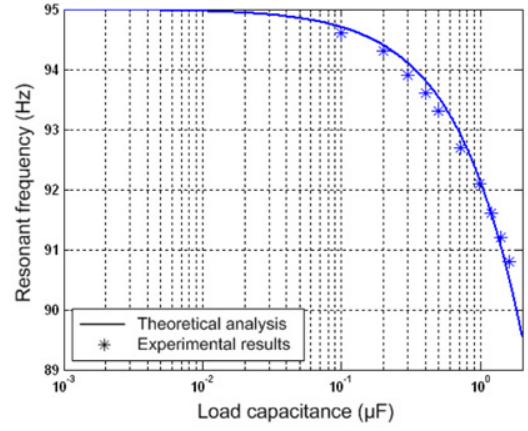

(a)

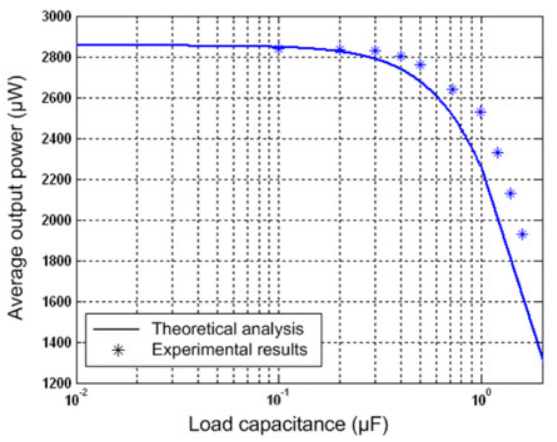

(b)

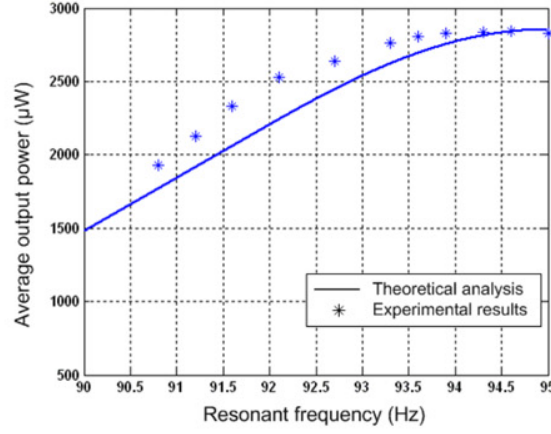

(c)

Figure 18. Tuning range and output power of MaETEM with variations of load capacitances (excited at $25 \mathrm{~m} g$ ). (a) $f_{\mathrm{r}}$ versus $C_{\mathrm{L}}$. (b) $P$ versus $C_{\mathrm{L}}$. (c) $P$ versus $f_{\mathrm{r}}$.

Table 3. Values used in equations (13) and (14) to obtain the theoretical curves in figure 18.

\begin{tabular}{|c|c|c|c|c|c|c|c|}
\hline $\begin{array}{l}K \\
(\mathrm{~T} \mathrm{~m})\end{array}$ & $\begin{array}{l}\text { Untuned resonant } \\
\text { frequency }(\mathrm{Hz})\end{array}$ & $m(\mathrm{~g})$ & $Q$ & $R_{\mathrm{c}}(\Omega)$ & $R_{\mathrm{L}}(\Omega)$ & $r$ & $a(\mathrm{~m} g)$ \\
\hline 552.25 & 95.5 & 830 & 1250 & 99.5 & 4000 & 40.2 & 25 \\
\hline
\end{tabular}

Table 4. Comparisons of performance of MiETEM and MaETEM.

\begin{tabular}{lccclll}
\hline & Mass $(\mathrm{g})$ & $\begin{array}{l}\text { Volume } \\
\left(\mathrm{cm}^{3}\right)\end{array}$ & $K(\mathrm{~T} \mathrm{~m})$ & $\begin{array}{l}\text { Effective tuning } \\
\text { range }(\mathrm{Hz})\end{array}$ & $\begin{array}{l}\text { Tuning } \\
\text { efficiency }\end{array}$ & $\begin{array}{l}\text { Maximum load } \\
\text { capacitance }^{\mathrm{b}}(\mathrm{nF})\end{array}$ \\
\hline MiETEM & 5 & 11 & 0.0035 & 0.15 & 0.27 & 1500 \\
\hline MaETEM & \multirow{2}{*}{830} & 289 & 552.25 & $\begin{array}{l}3.5 \\
>5\end{array}$ & $\begin{array}{l}3.68 \\
>5.24\end{array}$ & $>1400$ \\
\hline
\end{tabular}

a The ratio of the effective tuning range to the untuned resonant frequency.

$\mathrm{b}$ The maximum load capacitance that keeps the resonant frequency in the effective tuning range.

expected. When the load capacitance was small $(<100 \mathrm{nF})$, the maximum power output remained unchanged while the resonant frequency reduced. The maximum power output started decreasing as the load capacitance increased $(>100 \mathrm{nF})$. The experimental results of the resonant frequency agree with the theory while the experimental results show the maximum output power is slightly higher than the theory suggests, when excited at $25 \mathrm{mg}$. The reason is that there is a hard core inside the two coils and the coil inductance varies according to the change in magnetic flux within the hard core. The coil inductance of $4 \mathrm{H}$ was measured when the energy harvester was static. When the vibration level is small (e.g. $10 \mathrm{mg}$ ), the coil inductance does not change significantly. Thus the value of the capacitor required to cancel the effect of the coil inductance is close to theory based on the static situation. Therefore, the experimental results agree with the theoretical analysis when the excitation level is $10 \mathrm{mg}$. As the vibration level increases, the value of the capacitor required to cancel the effect of the coil inductance coil is different from the calculated value based on the static situation. The effect of the coil inductance on the performance of the energy harvester becomes apparent and therefore causes the mismatch between the experimental results and the theoretical analysis. Furthermore, the slight non-linearity of the energy harvester is another cause of the mismatch.

\section{Comparisons of the two electrically tunable energy harvesters}

Table 4 summarizes the performance of the two energy harvesters presented in sections 4 and 5 . It is found that the macroenergy harvester, MaETEM, has a larger effective tuning range than the microenergy harvester, MiETEM. The volume and mass of MaETEM are 166 and 26 times larger than those of the energy harvester MiETEM, respectively. The electromagnetic coupling factor of MaETEM is over 150000 times greater than that of MiETEM, which is the reason why MaETEM has a larger effective tuning range, as theory indicates.

In the microenergy harvester, MiETEM, the magnetic flux does not change while the inertial mass is vibrating. Only the magnetic flux through the coil changes. In the macroenergy harvester, MaETEM, the magnetic flux is changed by varying the reluctances of the air gaps. As the reluctances of the air gaps are much larger than those of magnetic flux guides, the reluctances of the air gaps dominate the total reluctance in the magnetic circuit. Small changes in the reluctance of air gap can significantly change the magnetic flux. Therefore, the magnetic flux change in MaETEM is much greater than that in MiETEM. As both energy harvesters have similar maximum displacements (around $0.2 \mathrm{~mm}$ ), the 
magnetic flux change per unit displacement in MaETEM is much greater than that in MiETEM. This is the reason why the electromagnetic coupling factor of MaETEM is much higher than that of MiETEM and thus MaETEM has a larger effective tuning range than MiETEM.

\section{Conclusions}

This paper presents a general model of resonant frequency tuning of vibration electromagnetic energy harvesters by varying the electrical load, specifically the capacitive load. It was found by theoretical analysis that a high electromagnetic coupling factor, $K$, must be achieved to realize a large tuning range. A large coil resistance results in a large tuning range with small load capacitances. The bigger the energy harvester, the higher $K$ must be to keep the output power of the energy harvester unchanged. To achieve a large $K$, the coil must have as high as possible fill factor and thickness. Furthermore, the layout of the magnets must be well designed so that the maximum change in magnetic field strength can be achieved with the minimum displacement of the magnets. The ratio of load resistance to coil resistance and the $Q$-factor should be as low as possible to achieve a high effective tuning range. The coil resistance does not affect the effective tuning range of the electromagnetic energy harvester. Finally, the inertial mass must be large to ensure high output power if $K$ is high. Therefore, electrical resonant frequency tuning is more feasible in macroenergy harvesters than in microenergy harvesters.

This model has also been validated by experiments. Resonant frequencies of a microelectromagnetic energy harvester as well as a macroelectromagnetic energy harvester were successfully tuned by varying their capacitive load. The micro-scale MiETEM had an untuned resonant frequency of $70.05 \mathrm{~Hz}$. The electromagnetic coupling factor of MiETEM was 0.0035 . It was tuned by $0.23 \mathrm{~Hz}$ while changing its capacitive load from 0 to $4000 \mathrm{nF}$ when excited at $30 \mathrm{mg}$. It had an effective tuning range of $0.15 \mathrm{~Hz}$ while its capacitive load was varied from 0 to $1500 \mathrm{nF}$. The macro-scale MaETEM had untuned resonant frequencies of $95.1 \mathrm{~Hz}$ and $95.5 \mathrm{~Hz}$ when excited at $10 \mathrm{mg}$ and $25 \mathrm{mg}$, respectively. The electromagnetic coupling factor of MaETEM was 552.25. It was tuned by $3.8 \mathrm{~Hz}$ by changing its capacitive load from 0 to $1400 \mathrm{nF}$ when excited at $10 \mathrm{mg}$. It had an effective tuning range of $3.5 \mathrm{~Hz}$ while its capacitive load was varied from 0 to $1200 \mathrm{nF}$. When the energy harvester was excited at $25 \mathrm{mg}$, its resonant frequency has been tuned by $4.2 \mathrm{~Hz}$ by changing its capacitive load from 0 to $1400 \mathrm{nF}$. The test results were found to agree well with the theoretical analysis to within $10 \%$.

Experimentally, the macro-scale MaETEM has a larger effective tuning range than the micro-scale MiETEM. The reason is that in MiETEM, the magnetic flux does not change as the inertial mass is vibrating and only the magnetic flux cutting the coil is changed, whereas in the macroenergy harvester MaETEM, the magnetic flux is changed by varying the reluctances of the air gaps. As the reluctances of the air gaps are much larger than those of magnetic flux guides, the reluctances of the air gaps dominate the total reluctance in the magnetic circuit. Small changes in reluctance of the magnetic flux guide have little effect on the magnetic flux in the circuit while small changes in the reluctance of air gap can significantly change the magnetic flux. Since the maximum displacements of both energy harvesters are similar, the magnetic flux change per unit displacement in MaETEM is much larger than that in MiETEM. Therefore, the macroenergy harvester MaETEM has much greater electromagnetic coupling factor than MiETEM and thus a larger effective tuning range. This also agrees with the key points concluded in section 3.3 for designing electromagnetic energy harvesters capable of frequency tuning using electrical methods.

In conclusion, in order to design an electrically tunable electromagnetic energy harvester with a larger effective tuning range, it is preferred that the energy harvester has a magnetic flux linkage whose air gap varies during operation. However, the material of the magnets and the magnetic flux guide should be carefully selected for this type of energy harvester. If the magnet is strong and the magnetic flux guide has very high permeability, there will be a strong magnetic force in the small air gap between the resonator and the stator, which will cause high non-linearity of the energy harvester. Furthermore, if this magnetic force is too strong, the movement of the resonator can be seriously restricted. This has to be taken into consideration when designing such electrically tunable electromagnetic energy harvesters.

\section{References}

[1] Beeby S and White N 2010 Energy Harvesting for Autonomous Systems (Boston, MA: Artech House Publishers) ISBN: 1596937181, 9781596937185

[2] Beeby S P, Tudor M J and White N M 2006 Energy harvesting vibration sources for microsystems applications Meas. Sci. Technol. 17 175-95

[3] Zhu D, Tudor M J and Beeby S P 2010 Strategies for increasing the operating frequency range of vibration energy harvesters: a review Meas. Sci. Technol. 21022001

[4] Sari I, Balkan T and Kulah H 2007 A wideband electromagnetic micro power energy harvester for wireless microsystems Proc. Int. Solid-State Sensors, Actuators and Microsystems Conf. 2007 (Lyon, June 2007) pp 275-8

[5] Tvedt L G W, Nguyen D S and Halvorsen E 2010 Nonlinear behavior of an electrostatic energy harvester under wideand narrowband excitation J. Microelectromech. Syst. $19305-16$

[6] Ferrari M, Ferrari V, Guizzetti M, Andò B, Baglio S and Trigona C 2010 Improved energy harvesting from wideband vibrations by nonlinear piezoelectric converters Sensors Actuators A 162 425-31

[7] Mann B P and Owens B A 2010 Investigations of a nonlinear energy harvester with a bistable potential well J. Sound Vib. 329 1215-26

[8] Ayala Garcia I, Zhu D, Tudor J and Beeby S 2010 A tunable kinetic energy harvester with dynamic over range protection Smart Mater. Struct. 19115005

[9] Eichhorn C, Tchagsim R, Wilhelm N and Woias P 2011 A smart and self-sufficient frequency tunable vibration energy harvester J. Micromech. Microeng. 21104003

[10] Zhu D, Roberts S, Tudor J and Beeby S 2010 Design and experimental characterization of a tunable vibration-based electromagnetic micro-energy harvester Sensors Actuators A 158 284-93 
[11] Wu X, Lin J, Kato S, Zhang K, Ren T and Liu L 2008 A frequency adjustable vibration energy harvester PowerMEMS 2008 (Sendai, Nov. 2008) pp 245-8

[12] Peters C, Maurath D, Schock W, Mezger F and Manoli Y 2009 A closed-loop wide-range tunable mechanical resonator for energy harvesting systems J. Micromech. Microeng. 19094004

[13] Eichhorn C, Tchagsim R, Wilhelm N,

Goldschmidtboeing F and Woias P 2010 A compact piezoelectric energy harvester with a large resonance frequency tuning range Proc. PowerMEMS 2010 (Leuven, Dec., 2010) pp 207-11
[14] Charnegie D 2007 Frequency tuning concepts for piezoelectric cantilever beams and plates for energy harvesting $M S c$ Dissertation School of Engineering, University of Pittsburgh, USA

[15] Cammarano A, Burrow S G, Barton D A W, Carrella A and Clare L R 2010 Tuning a resonant energy harvester using a generalized electrical load Smart Mater. Struct. 19055003

[16] Williams C B, Shearwood C, Harradine M A, Mellor P H, Birch T S and Yates R B 2001 Development of an electromagnetic micro-generator IEE Proc. Circuits Devices Syst. 148 337-42 